\title{
A UNIFIED EULERIAN THEORY OF TURBULENT DEPOSITION TO SMOOTH AND ROUGH SURFACES
}

\author{
Abhijit Guha* \\ Whittle Laboratory, University of Cambridge, Madingley Road, Cambridge CB3 ODY, U.K.
}

(First received 2 March 1995; and in final form 19 March 1997)

\begin{abstract}
This paper presents a simple, unified theory of deposition that is applicable for particles of any size, and reproduces very closely experimentally measured variation in deposition velocity with particle relaxation time. Apart from providing physical insight, the theory offers a simple, fast and reliable computational tool of practical use to aerosol engineers. The predictions are at least as accurate as the state-of-the-art particle-tracking calculations but require much less computational time. The theory includes the effects of thermophoresis, turbophoresis, electrostatic forces, gravity, lift force and surface roughness. The theory consists of writing the particle continuity and momentum conservation equations in their proper form and then performing Reynolds averaging. This procedure results in an expression for the particle flux which consists of three distinct terms for each of which a clear physical interpretation is available. The first term is a diffusive flux due to Brownian motion and turbulent fluctuation, the second is a diffusive flux due to temperature gradient (thermophoresis), and the third is a convective flux that arises primarily as an interaction between particle inertia and the inhomogeneity of the fluid turbulence field (turbophoresis). The lift force and electrostatic forces also contribute to this convective flux. It is shown that it is crucial to include the particle momentum equation in the analysis as this gives an estimate of the mentioned convective slip velocity of the particles. Absence of this equation in many previous studies which included only the particle continuity equation necessitated postulations such as stopping distance models. Only the dominant terms in the continuity and momentum equations are retained in the present analysis which give almost the same answer as with a calculation retaining all terms, but the former is more amenable to direct physical interpretation. The method of Reynolds averaging is general, and other effects not included in this study, e.g. pressure diffusion can easily be incorporated by including the appropriate forces in the particle momentum equation. The present study includes the effects of surface roughness, and the calculations show that the presence of small surface roughness even in the hydraulically smooth regime significantly enhances deposition especially of small particles. Thermophoresis can have equally strong effects, even with a modest temperature difference between the wall and the bulk fluid. For particles of the intermediate size range, turbophoresis, thermophoresis and roughness are all important contributors to the overall deposition rate. (C) 1997 Elsevier Science Ltd. All rights reserved
\end{abstract}

\section{INTRODUCTION}

Understanding the mechanisms by which particles dispersed in a turbulent stream of fluid are transported towards the solid walls forming the flow passage, and predicting the rate of deposition are both scientifically interesting and of engineering importance (in a variety of areas of mechanical engineering, chemical engineering and physiology). Consequently, these have been the subject-matter of an extremely large number of studies.

In the present paper, a simple, unified Eulerian theory of deposition has been developed, which can be used for practical calculations. Starting from the fundamental conservation equations and with a modicum of approximations and no tuning factors, the present theory produces satisfactory agreement with the experimental data (schematically shown in Fig. 1).

There are two common approaches for deposition calculations: Eulerian and Lagrangian. On the Eulerian front, the established practice has been to use separate theories for capturing the different behaviours of deposition rate in different size ranges of particles. The Lagrangian scheme, on the other hand, involves trajectory calculations typically for a large

\footnotetext{
* Present address: Aerospace Engg. Dept., University of Bristol, University Walk, Bristol BS8 1TR, U.K.
} 


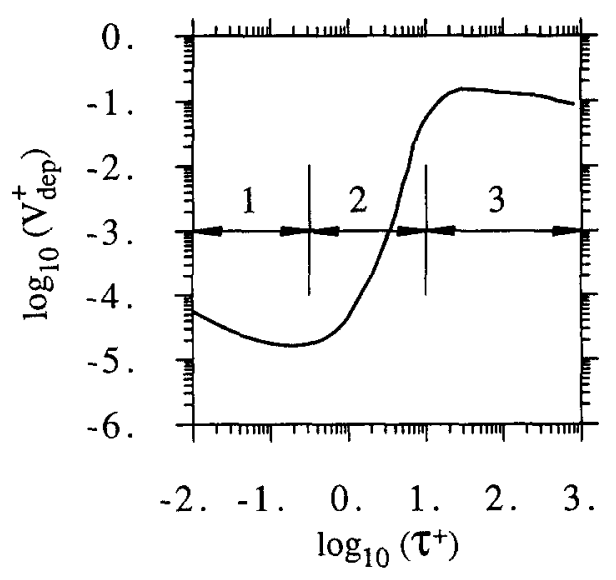

Fig. 1. A typical variation in measured deposition rate with particle relaxation time. Zone 1: turbulent diffusion regime; zone 2: turbulent diffusion -- eddy impaction regime; zone 3: particle inertia moderated regime.

number of particles, the fluid turbulence field being generated by various methods ranging from simple to direct numerical simulation (DNS) of Navier-Stokes equations. The present theory is Eulerian in nature but results in a universal set of simple equations that apply for the whole size range of particles and explain the physics of the problem to a great extent. The present scheme has the potential of becoming a useful tool in practical calculations of any complexity, as well as theoretical analyses of fundamental importance.

The present theory of deposition is general. Other than Fickian diffusion (both Brownian and turbulent), the theory includes motion of particles due to temperature gradient (thermophoresis), motion arising from the interaction of particle inertia with the inhomogeneity of turbulence field (turbophoresis), motion of particles due to electrical forces (electrophoresis), motion due to gravity and Saffman lift force, and the effects of surface roughness. It is possible to extend the theory to include other effects such as pressure diffusion, stressphoresis or diffusiophoresis.

The theory is mathematically quite sound and should be useful in understanding the physics of deposition process. However, one of the main strength of the theory is its simplicity and practical relevance. It is shown, for example, in Fig. 9 that the solution of a simple set of equations, the continuity equation (16) and two momentum equations (18a) and $(18 \mathrm{~b})$, produce the whole of the "S-shaped" deposition curve and give results which compare favourably with the most advanced and elaborate Lagranginan-type particle tracking methods (e.g. McLaughlin, 1989; Fichman et al., 1988; Fan and Ahmadi, 1993). (In fact, it is shown that the solution of just one momentum equation (18c) could be enough in many cases, the compromise being the neglect of the Saffman lift force.)

The present scheme, being Eulerian, is computationally much faster than stochastic Lagrangian calculations of particle tracking (by several order of magnitude in case of small particles). It is also more versatile than any other reported calculation scheme (in its applicability to the whole size range of particles and its ability to account for various deposition mechanisms). Since the present theory models the various deposition mechanisms correctly, it should be applicable to practical deposition problems in complex geometries (e.g. deposition of particles on internally cooled, highly curved, gas turbine blades or that of water droplets on steam turbine blades). It is possible to combine the present Eulerian scheme for calculating particle motion with well-established Eulerian flow solvers for calculating the flow field of the primary fluid. Thus, the present theory could be of interest to fluid dynamicists as well as of use to aerosol engineers.

The theory is applicable to dilute mixtures, i.e. when the volume fraction of the dispersed phase is low. The analysis is presented for two dimensional flow field, but it is straightforward to extend it to three dimensions or to axi-symmetric geometry. Although we have 
shown how to account for terms like virtual mass and Basset-Boussinesq force, we have restricted the further analysis to high relative density of the particulate material for which these terms become negligible. For all numerical illustrations and for comparisons with experiments and other theoretical predictions, we have considered the most explored flow configuration: vertical, fully developed pipe flow. However, the method is applicable to other flow configurations such as the boundary-layer-type flow on a predominantly horizontal surface.

A particular turbulence model has been used here (Section 2.5) for illustration, but the present theory is not limited on this account. Any other suitable turbulence model for describing the fluid flow field could be used in conjunction with the present theory of deposition.

\subsection{Representation and historical perspectives}

Usually the results of deposition experiments (e.g. Liu and Agarwal, 1974) or calculations are presented as curves of non-dimensional deposition velocity versus non-dimensional particle relaxation time. The deposition velocity, $V_{\text {dep }}$, is the particle mass transfer rate, $J$, normalized by the mean or bulk concentration, $c_{0}$, in the flow

$$
V_{\text {dep }}=J / c_{0} .
$$

The particle relaxation time, $\tau$, is a measure of particle inertia and denotes the time scale with which any slip velocity between the particles and the fluid is equilibrated. In the Stokes drag regime $\tau$ is given by

$$
\tau=2 \rho_{\mathrm{p}}^{\circ} r^{2} / 9 \mu
$$

where $\rho_{\mathrm{p}}^{\mathrm{o}}$ is the density of pure particulate material, $r$ is the radius of the particle and $\mu$ is the dynamic viscosity of the fluid.

We have plotted the results with this definition of relaxation time to be consistent with other works in the field. However, in the numerical calculations we have corrected the relaxation time to account for the slip velocity for large particles and rarefied gas effects for very small particles. The general expression (Guha, 1995) used is given by

$$
\tau_{\mathrm{I}}=\tau[\phi(\mathrm{Re})+4.5 \mathrm{Kn}],
$$

where $\phi(\operatorname{Re})$ is an empirical correction for large slip Reynolds numbers $(\operatorname{Re}=2 r|\Delta V| / v)$ given by $\phi(\operatorname{Re})=\left[1+0.15 \mathrm{Re}^{0.687}\right]^{-1}$ where $\Delta V$ is the slip velocity between the two phases and $v$ is the kinematic viscosity of the fluid. $\mathrm{Kn}$ is the Knudsen number defined by $\mathrm{Kn} \equiv \lambda / 2 r$ where $\lambda$ is the mean free path. The simple kinetic theory expression for $\lambda[\lambda=\mu \sqrt{ }(2 \pi \mathrm{R} T) / 2 p$, where $T$ is the temperature and $p$ is the pressure $]$ is used. For small slip Reynolds numbers and continuum flow $(\mathrm{Re} \ll 1, \mathrm{Kn} \ll 1)$ equation $(2 \mathrm{~b})$ reduces to the Stokes drag formula for a sphere. For free molecule flow $(\mathrm{Kn} \gg 1)$ an expression derivable from kinetic theory is obtained. The expression within the bracket in equation ( $2 \mathrm{~b}$ ) provides a simple interpolation formula for intermediate Knudsen numbers. The method of analysis is not dependent on the form of equation ( $2 b$ ), however, and other, possibly more suitable, expressions could easily be incorporated if desired.

$V_{\text {dep }}$ and $\tau$ are made dimensionless with the aid of the fluid friction velocity $u_{*}$.

$$
\begin{aligned}
V_{\mathrm{dep}}^{+} & \equiv V_{\mathrm{dep}} / u_{*}, \\
\tau^{+} & \equiv \frac{\tau u_{*}^{2}}{v}=\frac{2}{9}\left(\frac{\rho_{\mathrm{p}}^{0}}{\rho_{\mathrm{f}}}\right) \frac{r^{2} u_{*}^{2}}{v^{2}},
\end{aligned}
$$

where $\rho_{\mathrm{f}}$ is the fluid density.

Figure 1 shows a typical, schematic plot of $V_{\text {dep }}^{+}$versus $\tau^{+}$as obtained by experiments. The results fall in three distinct categories. At first, as $\tau^{+}$increases, the deposition velocity 
decreases. This is the so-called "turbulent diffusion regime", where a turbulent version of Fick's law of diffusion applies. For $0.1<\tau^{+}<10$, the diffusion velocity increases with $\tau^{+}$. This is the so-called "eddy diffusion-impaction regime". The striking feature of this regime is that the deposition velocity increases by three to four order of magnitude. Separate models representing additional mechanisms of deposition had to be developed in order to explain this behaviour of deposition velocity. One of the most used calculation methods is called the "free flight" or "stopping distance" model (e.g. Friedlander and Johnstone, 1957, Davies, 1966, Beal, 1970).

In all of these models it is assumed that particles diffuse to within one "stopping distance", $s$, from the wall, at which point they make a "free flight" to the wall. The main difference between different models of this type lies in prescribing the free-flight velocity, $v$. Davies assumed that the free-flight velocity, $t$. is the same as the local RMS velocity of the fluid, but he obtained poor agreement with the experiments. (Liu and Ilori, 1974, improved the prediction of this model by prescribing, rather arbitrarily, a particle diffusivity which was different from the commonly used eddy momentum diffusivity.) Friedlander and Johnstone (1957), on the other hand, assumed $v=0.9 u_{*}$, a value close to the fluid RMS velocity in the outer layer. Their model agrees well with the experiments. Beal (1970) gave another variation of the stopping distance model.

It may be seen that these models are physically not very satisfactory, and predict monotonic rise in stopping distance (which may exceed the buffer layer thickness for larger particles, as $s^{+} \sim \tau^{+}$) and deposition velocity with increasing $\tau^{+}$. Experiments, however, show a third regime of deposition, usually termed "particle inertia moderated regime", in which the deposition velocity decreases with further increase in $\tau^{+}$(see Fig. 1). Stopping distance models are not of much use here, and new theories (e.g. Reeks and Skyrme, 1976) need to be applied.

Thus, even for the apparently simple case of turbulent deposition in a fully developed pipe flow, the usual theories are not in a very satisfactory state. The common practice is to use separate theories for each of the deposition regimes. Although it is possible, with proper tuning of the models (e.g. by prescribing the free-flight velocity), to reproduce the experimental results for fully developed pipe flow, the theories cannot be extrapolated to two- or three-dimensional flow situations (e.g. for deposition of particles on gas turbine blades) with any great confidence because of their piecemeal nature and the required empirical tuning. These models are also of limited use if other effects, e.g. thermophoresis or electrostatic interaction, are present.

A large number of recent studies have adopted the alternative approach of particle tracking in a Lagrangian framework. For example, Kallio and Reeks (1989) calculated the deposition of particles in a simulated turbulent fluid field; Ounis et al. (1993), and, Brooke et al. (1994) computed the motion of particles where the fluid motion was determined by direct numerical solution of the Navier-Stokes equations; and the calculations of Fichman et al. (1988), and, Fan and Ahmadi (1993) were based on the sublayer approach originally proposed by Cleaver and Yates (1975). These calculations are illustrative and important for physical understanding. However, Lagrangian computations typically involve the determination of trajectories of a very large number of particles (to establish statistically meaningful average quantities) and may be too time consuming to be effective as a practical calculation method, especially for small particles. (Many reported Lagrangian schemes also do not reproduce the whole deposition curve. The Kallio and Reeks model, for example, is not applicable in zone 1 of Fig. 1.) For example, small particles, say with non-dimensional relaxation time $\tau^{-}=1$, require CPU times for a Lagrangian simulation that may easily be three order of magnitude higher than the CPU time needed by the present Eulerian scheme A Lagrangian simulation for $\tau^{+}<1$ may take prohibitively large CPU time for practical applications.

In this paper, we, therefore, develop a simple, Eulerian theory that is applicable to all regimes of deposition. This approach identifies and models the actual mechanisms of deposition and is physically satisfying. We have summarized the special features of the present theory in greater detail towards the end of Section 3 and in Section 4. 


\section{THE PRESENT MODEL OF DEPOSITION}

The proper way of deriving the equations for deposition is to write the particle continuity and the momentum conservation equations, split the different flow quantities into their respective mean and fluctuating components, and then perform Reynolds averaging. If the fluid flow field is known, the Reynolds averaged particle conservation equations would specify the motion of the particles. It is crucial to include the particle momentum conservation equation in the analysis. Failure to do so in many previous studies has resulted in empirical hypotheses in the deposition models (e.g. the stopping distance models) in order to match theoretical predictions with experimental data.

However, before presenting the rigorous analysis, we would first take a physical, rather intuitive, approach in order to provide qualitative understanding.

\subsection{Modelling turbulent and molecular diffusion}

The deposition of small particles in a turbulent boundary layer is usually calculated by integrating a modified Fick's law of diffusion

$$
J=-\left(D_{\mathbf{B}}+D_{\mathrm{t}}\right) \frac{\mathrm{d} c}{\mathrm{~d} y},
$$

where $D_{\mathrm{B}}$ is the Brownian diffusivity, $D_{\mathrm{t}}$ is the turbulent diffusivity which varies with position, $y$ is the perpendicular distance from the wall, and $\mathrm{dc} / \mathrm{d} y$ is the concentration gradient. It is assumed that the mass diffusivity of the particles due to turbulence, $D_{t}$, is same as the eddy viscosity of the fluid, $\varepsilon . D_{\mathrm{B}}$ is given by the Einstein equation incorporating the Cunningham correction for rarefied gas effects,

$$
D_{\mathrm{B}}=\frac{k T(1+2.7 \mathrm{Kn})}{6 \pi \mu r},
$$

where $k$ is the Boltzmann constant, $T$ is the absolute temperature and $\mathrm{Kn}$ is the Knudsen number defined by $\mathrm{Kn} \equiv \lambda / 2 r$ where $\lambda$ is the mean free path of the surrounding gas and $r$ is the radius of a particle.

It is profitable to non-dimensionalize equation (4) such that

$$
V_{\mathrm{dep}}^{+}=-\left(\frac{D_{\mathrm{B}}}{v}+\frac{\varepsilon}{v}\right) \frac{\mathrm{d} c^{+}}{\mathrm{d} y^{+}},
$$

where

$$
c^{+}=c / c_{0}, \quad y^{+}=y u_{*} / v .
$$

In the present work we use the universal expression of eddy viscosity valid for all $y^{+}$, as given by Davies (1966)

$$
\frac{\varepsilon}{v}=y^{+\left(4-y^{+0.08}\right)}\left[\frac{2.5 \times 10^{7}}{\operatorname{Re}}\right]^{\left(-y^{+} /\left(400+y^{+}\right)\right)} \times 10^{-3},
$$

where $\mathrm{Re}$ is the Reynolds number based on the average velocity in the pipe.

From equations (3) and (5) it can be seen that the particular combination of the dimensionless variables $\left(D_{\mathrm{B}} / v\right)^{2 / 3}\left(\tau^{+}\right)^{1 / 3}$ is independent of the particle radius (for large particle radius when the terms involving $\mathrm{Kn}$ representing rarefied gas effects can be neglected), and, depends only on the flow condition. For example, in the case of flow in a small pipe of radius $R$, it can be shown (using Blasius's formula) that

$$
\left(\frac{D_{\mathrm{B}}}{v}\right)^{2 / 3} \tau^{+1 / 3}=0.01835\left(\frac{k T}{\rho_{\mathrm{f}} \nu^{2} R}\right)^{2 / 3}\left(\frac{\rho_{\mathrm{p}}^{\mathrm{o}}}{\rho_{\mathrm{f}}}\right)^{1 / 3}(\mathrm{Re})^{14 / 24}=\psi .
$$

For a particular $\psi$, corresponding to a specific flow condition, $D_{\mathbf{B}} / v$ decreases with increasing $\tau^{+}$. Hence the deposition velocity, $V_{\text {dep }}^{+}$, given by equation (6) always decreases with increasing $\tau^{+}$. Experiments, however, tell quite a different story (Fig. 1). 


\subsection{Motion of particles in inhomogeneous turbulent field}

Therefore, Fick's law of diffusion, as given by equation (4), does not encapsulate the whole physics of deposition and neglects a mechanism, operative in inhomogeneous turbulent flow that assumes dominance for large particle relaxation times. For a qualitative understanding refer to Fig. 2a and b. Fig. 2a shows the effect of a concentration gradient in homogeneous turbulence. Small particles almost faithfully follow the fluid eddies. At any location, the probability of a particle getting transported by a fluid eddy to the left is the same as that to the right. However, because there is a greater number of particles further away from the wall, the flux crossing any imaginary cross-sectional plane from the right exceeds that from the left. Consequently, there is a net flux of particles towards the wall against the concentration gradient. (Similar explanations are valid for particle flux due to Brownian motion as well.) This process of deposition is modelled by the turbulent version of Fick's law of diffusion.

Now consider Fig. $2 b$ which depicts a uniform concentration of particles in an inhomogeneous turbulent flow. In this configuration there is a gradient of turbulence intensity perpendicular to the wall. The particles are assumed large to have considerable inertia so that they may slip through the containing fluid eddy. At any particular location, the probability of a fluid eddy throwing a particle towards the right is the same as that of a fluid eddy throwing a particle towards the left. However, the probability of a particle being thrown rightward from a region of low turbulence intensity so that it reaches the imaginary plane is less than that of a particle being thrown leftward from a region of high turbulence intensity. Consequently, there is a net flux of particles from a region of high turbulence intensity to a region of low turbulence intensity. This transfer of mass against a gradient in turbulence intensity is termed "turbophoresis" (Reeks, 1983). (More qualitative and

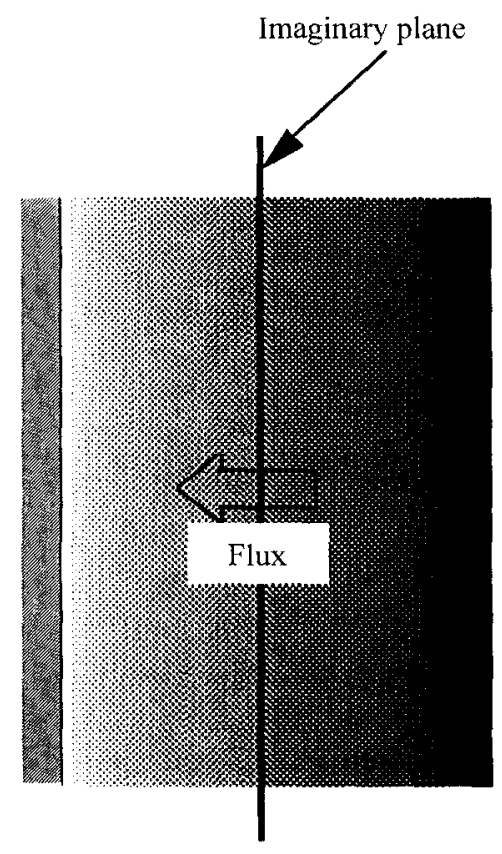

Increasing concentration

(a)

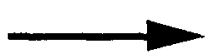

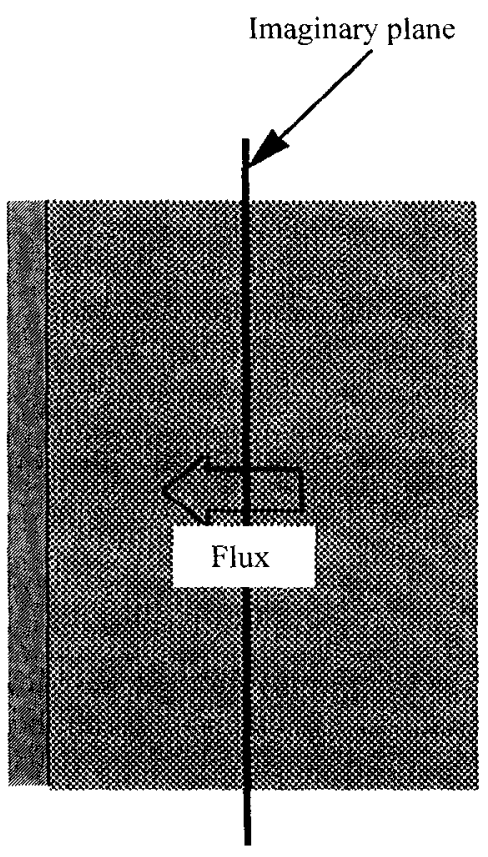

Increasing turbulence intensity

(b)

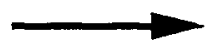

Fig. 2. Schematic diagram showing two different mechanisms of deposition. (a) Flux of particles driven by concentration gradient; (b) flux of particles driven by a gradient in turbulence intensity or "turbophoresis" even though the concentration of particles is uniform. 
quantitative discussion on turbophoresis is given after equations (18).) To formulate a unified theory of turbulent deposition one, therefore, has to combine concentrationgradient-driven deposition (Fick's law) with turbophoresis.

\subsection{Unified mathematical model for deposition}

It turns out that such a uniform description automatically arises from the basic conservation equations of the fluid-particle system. We follow the approach of Ramshaw (1979) to describe the motion of a particle cloud in a flowing fluid. (Ramshaw presents a phenomenological approach. For a kinetic theory of the dispersed phase see, for example, Reeks 1992.) The particles are assumed to constitute a hypothetical ideal gas whose partial pressure $p_{\mathbf{p}}$ is given by

$$
p_{\mathrm{p}}=(k / m) \rho_{\mathrm{p}} T,
$$

where $m$ is the mass of an individual particle and $\rho_{\mathrm{p}}$ is the partial density. It is assumed, while writing equation (10), that the particles are in local thermal equilibrium with the surrounding fluid. This equilibrium is brought about by their collisions with the fluid molecules, the particles themselves rarely collide with each other. If $n$ is the number of particles per unit volume of the mixture, then $\rho_{\mathrm{p}}$ is given by

$$
\rho_{\mathrm{p}}=n m,
$$

$\rho_{\mathrm{p}}$ is the same as particle concentration, $c$, used in equation (4). The mixture is considered dilute. The motion of the hypothetical ideal gas consisting of the particles is governed by the continuity and the momentum equations of fluid dynamics. For simplicity, we assume that the radius of the pipe is large so that the effects of the curvature can be neglected and the governing equations can be profitably written in the Cartesian coordinate. In the steady state, the equations of motion are

$$
\begin{gathered}
\nabla \cdot\left(\rho_{\mathrm{p}} \mathbf{V}_{\mathbf{p}}\right)=0, \\
\rho_{\mathbf{p}}\left(\mathbf{V}_{\mathbf{p}} \cdot \nabla\right) \mathbf{V}_{\mathbf{p}}=-\nabla p_{\mathbf{p}}+\rho_{\mathbf{p}} \mathbf{F}+\rho_{\mathbf{p}} \mathbf{G},
\end{gathered}
$$

where the vector $\mathbf{V}_{\mathbf{p}}$ represents the mean velocity of the particles, on which is superposed a random thermal velocity that gives rise to the partial pressure $p_{\mathrm{p}}$. As a result of the equipartition of energy, the RMS thermal velocity of the particles is much less than that of the fluid molecules. [Young (1991) and Johansen (1991) performed Reynolds averaging, similar to what is presented below, on a form of continuity equation which, unlike equation (12), includes the diffusive fluxes explicitly. In Ramshaw's treatment diffusion arises through the momentum equation.] $\mathbf{F}$ is the mean force per unit mass on the particles due to the fluid and $\mathbf{G}$ is the total external force per unit mass (e.g. gravitational, electromagnetic) on the particles. $\mathbf{F}$ is given by (Ramshaw, 1979)

$$
\mathbf{F}=\left(\mathbf{V}_{\mathrm{f}}-\mathbf{V}_{\mathrm{p}}\right) / \tau_{\mathrm{I}}-(\eta / m) \nabla \ln T-\left(1 / \rho_{\mathrm{p}}^{\mathrm{o}}\right) \nabla p+\mathbf{F}_{\mathrm{v}}+\mathbf{F}_{\mathrm{B}}+\mathbf{F}_{\mathrm{S}}
$$

where, $\mathbf{V}_{f}$ is the fluid velocity, $\rho_{\mathrm{p}}^{\mathrm{o}}$ is the mass density of the pure particulate material, $p$ is the true pressure of the fluid-particle system, $\eta$ is the thermophoretic force coefficient (Talbot et al., 1980). $\mathbf{F}_{\mathrm{v}}, \mathbf{F}_{\mathrm{B}}$ and $\mathbf{F}_{\mathrm{S}}$ are the virtual mass force, the Basset-Boussinesq force and the Saffman lift force, respectively. The term containing $\nabla p$ is the source of the buoyant force on a particle in a stationary fluid in a gravitational field. This term and $\mathbf{F}_{\mathrm{v}}$ and $\mathbf{F}_{\mathrm{B}}$ are usually small (because of the high material density, $\rho_{\mathrm{p}}^{\mathrm{o}}$, of the particles) compared with the first term on the RHS of equation (14) which represents the steady-state drag force. In the present study they are not included. The thermophoretic force may become appreciable for small particles if the temperature gradient is high (as is the case for internally cooled gas turbine blades) and is retained in the present analysis. The thermophoretic force coefficient, $\eta$, is 
given by (Talbot et al., 1980)

$$
\eta=\frac{2.34(6 \pi \mu v r)\left(\lambda_{\mathrm{r}}+4.36 \mathrm{Kn}\right)}{(1+6.84 \mathrm{Kn})\left(1+8.72 \mathrm{Kn}+2 i_{\mathrm{r}}\right)},
$$

where, $\lambda_{\mathrm{r}}$ is the ratio of the thermal conductivity of the fluid, $\lambda_{\mathrm{f}}$, and that of the particles, $\lambda_{\mathrm{p}}\left(\lambda_{\mathrm{r}}=\lambda_{\mathrm{f}} / \lambda_{\mathrm{p}}\right)$.

Now we consider a two-dimensional flow field and decompose the instantaneous flow parameters into their mean and fluctuating parts

$$
\begin{aligned}
& V_{\mathrm{f} x}=\bar{V}_{\mathrm{f} x}+V_{\mathrm{f} x}^{\prime}, \\
& V_{\mathrm{f} y}=\bar{V}_{\mathrm{f} y}+V_{\mathrm{f} y}^{\prime}, \\
& V_{\mathrm{p} x}=\bar{V}_{\mathrm{p} x}+V_{\mathrm{p} x}^{\prime}, \\
& V_{\mathrm{p} y}=\bar{V}_{\mathrm{p} y}+V_{\mathrm{p} y}^{\prime}, \\
& \rho_{\mathrm{p}}=\bar{\rho}_{\mathrm{p}}+\rho_{\mathrm{p}}^{\prime},
\end{aligned}
$$

where the suffix $x$ represents the respective components along the $x$-coordinate which is along the flow direction and the suffix $y$ represents the respective components in the direction perpendicular to the wall. The suffices $f$ and $p$ refer to the fluid and the particles, respectively. We now substitute equation (15) into equations (12) and (13), and take time mean of the resulting equations. The details of the procedure are given in Appendix A.

Johansen (1991) has presented a similar Reynolds-averaging analysis. The starting point of the present formulation has, however, been Ramshaw's (1979) equations of motion which are internally consistent and physically satisfying. It is also very easy to include new forces in Ramshaw's equations thereby extending the scope of the theory of deposition. We have then systematically Reynolds-averaged both the continuity and momentum equations simultaneously. Finally, with minor approximations, we have converted equations (A3), (A6) and (A7) into the equation set (16)-(18) which are far simpler in form and are amenable to direct physical interpretation. The present formulation also includes the effects of thermophoresis and roughness of the wall.

\subsection{Physical description of the model}

If the flux of particles in the $y$ direction (which is perpendicular to the solid wall) is denoted by $J$, then equation (A11) of the Appendix A shows that

$$
J=-\left(D_{\mathrm{B}}+\varepsilon\right) \frac{\partial \bar{\rho}_{\mathrm{p}}}{\partial y}-\bar{\rho}_{\mathrm{p}} D_{T} \frac{\partial \ln T}{\partial y}+\bar{\rho}_{\mathrm{p}} \bar{V}_{\mathrm{p} y}^{\mathrm{c}},
$$

where the coefficient of temperature-gradient-dependent diffusion, $D_{T}$, is given by

$$
D_{T}=D_{\mathrm{B}}(1+\eta / k T) \text {. }
$$

and includes the thermophoretic component. Equation (16) is the generalized equation for particle flux. Note, however, that we did not retain the pressure diffusion term and $\mathbf{F}_{\mathrm{B}}$ and $\mathbf{F}_{\mathrm{v}}$ of equation (14) for further analysis, and did not include, say, stressphoresis forces in equation (14). The particle convective velocity in the $y$ direction, $\bar{V}_{\mathrm{py}}^{\mathrm{c}}$, appearing in equation (16) has to be calculated from the particle momentum equation. The particle momentum equations in the $y$ and $x$ directions are given by equations (A9) and (A10), derived in Appendix A, which we reproduce here, in the same order, for the ease of reference.

$$
\begin{gathered}
\bar{V}_{\mathrm{py}}^{\mathrm{c}} \frac{\partial \bar{V}_{\mathrm{py}}^{\mathrm{c}}}{\partial y}+\frac{\bar{V}_{\mathrm{p} y}^{\mathrm{c}}}{\tau_{1}}=-\frac{\partial \overline{V_{\mathrm{p} y}^{\prime 2}}}{\partial y}+F_{\mathrm{S} y}+G_{\mathrm{E} y}, \\
\bar{V}_{\mathrm{p} y}^{\mathrm{c}} \frac{\partial \bar{V}_{\mathrm{p} x}}{\partial y}=\frac{1}{\tau_{1}}\left(\bar{V}_{\mathrm{i} x}-\bar{V}_{\mathrm{p} x}\right)+g .
\end{gathered}
$$


Equations (18a) and (18b) are specialized for fully developed, vertical flow. Note that the $x$-momentum equation (18b) involves both $\bar{V}_{\mathrm{p} x}$ and $\bar{V}_{\mathrm{p} y}^{\mathrm{c}}$. The $y$-momentum equation (18a), on the other hand, is almost decoupled and depends on $\bar{V}_{p x}$ only through the Saffman Lift force, $F_{S y}$. A study of equations (18a) and (18b) also shows nicely how gravity affects the $y$-momentum equation through the lift force. The LHS of equation (18b) involves $\bar{V}_{\mathrm{py}}^{\mathrm{c}}$. As a result of this convective velocity in the $y$-direction, the direction of the lift force may remain unaltered (Appendix B) whether the flow is vertically downwards or upwards [replace $g$ by $-g$ in equation (18b)], and the influence of gravity on the deposition velocity is likely not to be serious.

In the general case, both equations (18a) and (18b) must be solved simultaneously. This calculation is presented later (Figs 8 and 9) in order to reveal the effect of including the Saffman lift force. For the time being, in order to gain more physical insight into the deposition process, we neglect the effect of the Saffman Lift force, $F_{s y}$. With these provisos, the particle convective velocity in the $y$ direction, $\bar{V}_{p y}^{\mathrm{c}}$, can be calculated from

$$
\bar{V}_{\mathbf{p} y}^{\mathrm{c}} \frac{\partial \bar{V}_{\mathbf{p} y}^{\mathrm{c}}}{\partial y}+\frac{\bar{V}_{\mathrm{py}}^{\mathrm{c}}}{\tau_{\mathrm{l}}}=-\frac{\partial \overline{V_{\mathrm{p} y}^{\prime 2}}}{\partial y}\left(+G_{E y}\right)
$$

The electrical force, $G_{\mathrm{E} y}$, is not retained in the further analysis in order to keep the discussion focused. It is worth remembering that the second term in the LHS of equation $(18 \mathrm{c})$ is the steady state drag term simplified with the assumption $\bar{V}_{\mathrm{f} y}=0$; the full form is $-\left(\bar{V}_{\mathrm{f} y}-\bar{V}_{\mathrm{py} y}^{\mathrm{c}}\right) / \tau_{\mathrm{I}}$.

The first term in the RHS of equation (16) is the diffusion due to a gradient in the particle concentration [same as Fick's law given by equation (4)], the second term represents the diffusion due to a gradient in the temperature and the third term represents a convective contribution arising due to particle inertia. Equation (18c) relates the particle convective velocity with the gradient in turbulence intensity (turbophoresis). It is chiefly the absence of this convective term in Fick's law that necessitated postulating stopping distance models. It is important to note that the turbophoretic term depends on the particle RMS velocity, which may be different from the fluid RMS velocity if the particle inertia is large. When the particles are very small, they effectively follow the fluid eddies and the two RMS velocities are essentially the same. In this limit, $\tau_{\mathrm{I}} \rightarrow 0$. Equation $(18 \mathrm{c})$ shows that $\bar{V}_{\mathrm{p} y}^{\mathrm{c}} \rightarrow 0$. Hence, the contribution from turbophoresis is negligible. Fick's law is, therefore, an adequate description for the deposition of small particles. As $\tau_{\mathrm{I}}$ increases, the turbophoretic term assumes dominance, thereby increasing the deposition rate by a few order of magnitude. However, as $\tau_{1}$ increases, the particles are less able to follow fluid fluctuations and the particle RMS velocity becomes progressively smaller as compared to the fluid RMS velocity. This is one of the factors responsible for the eventual decrease in deposition velocity with increasing particle size when $\tau_{\mathrm{I}}$ is very large (see the fourth and fifth paragraphs from the end of Section 4).

Equation (18c) is almost exact and does not depend on the concentration profile. It is a first-order, non-linear differential equation. It can be written in finite difference form and integrated with one boundary condition. $\left[\bar{V}_{\mathbf{p y}}^{\mathbf{c}}=0\right.$ at the pipe centreline, or, at a sufficient distance away from the wall where the gradient in turbulence intensity is negligibly small. In a boundary-layer-type calculation, one could specify the "laminar slip velocity" at the edge of the boundary layer.] The convective velocity, $\bar{V}_{\mathrm{p} y}^{\mathrm{c}}$, can, therefore, be determined if the particle RMS velocity could be estimated properly. For not too large particles, the drag term (second term in LHS) almost balances the turbulence term (RHS), and, the acceleration term (first term in LHS) is negligible. The acceleration term assumes importance for large particles and should not be neglected. It should be noted from equation (18c) that $\bar{V}_{p y}^{\mathrm{c}}$ is always zero (or equal to the "laminar slip velocity") if the turbulent field is homogeneous.

The equation set (16)-(18) is almost exact and should work well if one could find an accurate expression for the particle RMS velocity as a function of the wall coordinate. The variation of fluid RMS velocity as a function of wall coordinate has been measured and is well established. We, therefore, relate the particle mean-square velocity to the fluid 
mean-square velocity through a parameter $\mathfrak{R}$ which is defined as the ratio of the two.

$$
\mathfrak{R}=\overline{V_{n y}^{\prime 2}} \overline{V_{f y}^{\prime 2}} \text {. }
$$

There is no definitive (accurate and yet simple) mathematical model for $\mathfrak{R}$, particularly, in an inhomogeneous turbulence field. In reality, there should be a memory effect by which the migrating particles tend to retain the turbulence levels of earlier instants. In a practical Eulerian-type calculation, however, estimates of $\mathfrak{R}$ are to be made from local turbulence properties. This may not be very accurate, especially since the gradient of fluid turbulence near the wall is very high. In defence of a simple calculation scheme, it may be noted however that (the not unsuccessful) mixing length theories of fluid turbulence employ similar assumptions.

Simple theories of homogeneous turbulence predict that for the particles to be in local equilibrium with the fluid turbulence $\Re=T_{\mathrm{L}} /\left(\tau_{1}+T_{\mathrm{L}}\right)$, where $T_{\mathrm{L}}$ is the Lagrangian time scale of fluid turbulence. Some measurements in this area are reported by Binder and Hanratty (1991) and Vames and Hanratty (1988). Binder and Hanratty provide the following experimental correlation for $\mathfrak{R}$ (their equation (18)):

$$
\mathfrak{R}=\frac{1}{1+0.7\left(\tau_{\mathrm{I}} / T_{\mathrm{L}}\right)} .
$$

We use, as a first step, equation (20) in the present calculations. $\mathfrak{R}$ varies with the wall co-ordinate as $T_{L}$ varies. For very small particles $\tau_{I} \rightarrow 0$, and consequently $\mathfrak{R} \rightarrow 1$. In other words, very small particles essentially follow fluid turbulence. For large particles, $\tau_{\mathrm{I}} \rightarrow \infty$, $\mathfrak{R} \rightarrow 0$.

We can non-dimensionalize equations (18c) and (16) as

where

$$
\begin{aligned}
& \bar{V}_{\mathbf{p y}}^{\mathrm{c}+} \frac{\partial \bar{V}_{\mathbf{p y}}^{\mathrm{c}+}}{\partial y^{+}}+\frac{\bar{V}_{\mathrm{py}}^{\mathrm{c}+}}{\tau_{\mathrm{l}}^{+}}=-\frac{\partial}{\partial y^{+}}\left(\mathfrak{\Re} \overline{V_{\mathrm{fy}}^{y^{+2}}}\right), \\
& V_{\mathrm{dep}}^{+}=-\left(\frac{D_{\mathrm{B}}}{v}+\frac{\varepsilon}{v}\right) \frac{\partial \rho_{\mathrm{p}}^{+}}{\partial y^{+}}-\rho_{\mathrm{p}}^{+} D_{\mathrm{T}}^{+} \frac{\partial \ln T}{\partial y^{+}}+\rho_{\mathrm{p}}^{+} \bar{V}_{\mathrm{p} y}^{\mathrm{c}+},
\end{aligned}
$$

$$
\begin{aligned}
V_{\mathrm{dep}}^{+} & =J /\left(\rho_{\mathrm{p}_{u}} u_{*}\right), \\
\bar{V}_{\mathrm{py}}^{\mathrm{c}+} & =\bar{V}_{\mathrm{py}}^{\mathrm{c}} / u_{*}, \\
\rho_{\mathrm{p}}^{+} & =\bar{\rho}_{\mathrm{p}} / \rho_{\mathrm{p}_{i}}, \\
V_{\mathrm{fy}}^{\prime+} & =V_{\mathrm{f} y}^{\prime} / u_{*}, \\
D_{T}^{+} & =D_{T} / v,
\end{aligned}
$$

and $\rho_{p_{0}}$ is the partial density (concentration) of the particles at the pipe centreline. Similar non-dimensionalization is possible for equations (18a) and (18b).

\subsection{Modelling fluid turbulence}

The mean motion of the fluid in the axial direction $\left(\vec{V}_{\mathrm{f} x}\right.$ and the RMS fluctuation in the $y$-direction $\left(\sqrt{\bar{V}_{\mathrm{f} y}+\overline{\bar{z}}}\right)$ are expressed as functions of the wall-coordinate $\left(y^{+}\right)$as given by Kallio and Reeks (1989) (with corrections of the errors in their paper).

Mean motion of the fluid in the axial direction $\left(V_{\mathrm{f} x}^{+} \equiv \bar{V}_{\mathrm{f} x} / u_{*}\right)$ :

$$
\begin{array}{ll}
V_{\mathrm{f} x}^{+}=y^{+} & \text {for } y^{+} \leqslant 5, \\
V_{\mathrm{f} x}^{+}=a_{0}+a_{1} y^{+}+a_{2} \mathrm{y}^{+2}+a_{3} y^{+3} & \text { for } 5<y^{+}<30, \\
V_{\mathrm{f} x}^{+}=2.5 \ln y^{+}+5.5 & \text { for } y^{+} \geqslant 30,
\end{array}
$$

where $a_{0}=-1.076, a_{1}=1.445, a_{2}=-0.04885, a_{3}=0.0005813$. 
Fluid RMS velocity in the $y$-direction $\left(\sqrt{V_{\mathrm{fy}}^{\prime 2}} \equiv \sqrt{V_{\mathrm{fy}}^{\prime 2}} / u_{*}\right)$ :

$$
\sqrt{V_{\mathrm{fy}}^{\prime+2}}=\frac{0.005 y^{+2}}{1+m y^{+n}} \quad \text { for } 0<y^{+}<200,
$$

where $m=0.002923, n=2.128$

Lagrangian time scale $\left(\mathrm{T}_{\mathrm{L}}^{+} \equiv T_{\mathrm{L}} u_{*}^{2} / v\right)$ :

$$
T_{\mathrm{L}}^{+}=\frac{\varepsilon}{v}\left(1 / \overline{\boldsymbol{V}_{\mathrm{f} y}^{\prime+2}}\right),
$$

where $\varepsilon$ is given by equation (8).

Temperature profile in the pipe (Kay and Nedderman, 1988):

$$
\begin{array}{ll}
\frac{T-T_{\mathrm{w}}}{\Delta T}=\frac{\operatorname{Pr} y^{+}}{\Delta T_{200}^{+}} & \text {for } y^{+}<5, \\
\frac{T-T_{\mathrm{w}}}{\Delta T}=\frac{5 \operatorname{Pr}+5 \ln \left(0.2 \operatorname{Pr} y^{+}+1-\operatorname{Pr}\right)}{\Delta T_{200}^{+}} & \text {for } 5 \leqslant y^{+} \leqslant 30, \\
\frac{T-T_{\mathrm{w}}}{\Delta T}=\frac{5 \operatorname{Pr}+5 \ln (1+5 \operatorname{Pr})+2.5 \ln \left(y^{+} / 30\right)}{\Delta T_{200}^{+}} & \text {for } 30<y^{+}<200,
\end{array}
$$

where $\Delta T_{200}^{+}=5 \operatorname{Pr}+5 \ln (1+5 \operatorname{Pr})+2.5 \ln (200 / 30), T$ is the local temperature of the fluid, $T_{\mathrm{w}}$ is the wall temperature, $\operatorname{Pr}$ is the Prandtl number of the fluid and $\Delta T$ is the temperature difference between $y^{+}=200$ and the wall.

Figure 3 shows the variation of diffusivity, fluid RMS velocity and the temperature profile as functions of wall coordinate.

\subsection{Extension of the unified deposition model to rough surfaces}

Following Wood (1981), we assume that, on a rough surface, the virtual origin of the velocity profile is shifted by a distance $e$ away from the wall such that $e=0.55 k_{\mathrm{s}}$, where $k_{\mathrm{s}}$ is the effective roughness height. We further assume that the particles are captured when they reach the level of effective roughness height, i.e. at a distance $b$ above the origin of the velocity profile, where $b=k_{\mathrm{s}}-e=0.45 k_{\mathrm{s}}$. Figure 4 schematically shows the different parameters, e.g. $k_{\mathrm{s}}, b$ and $e$.

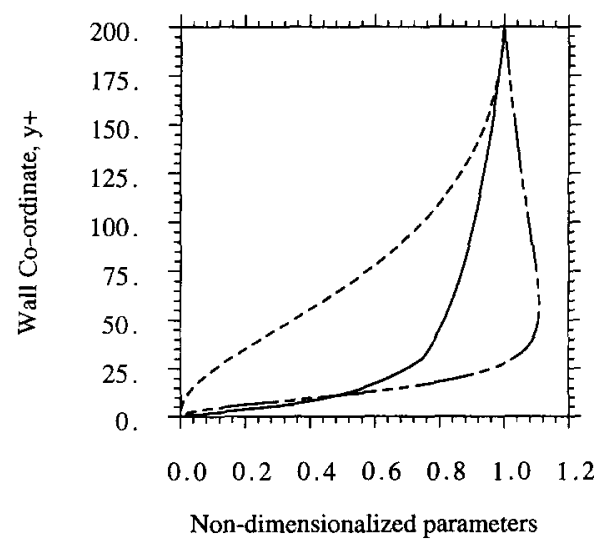

Fig. 3. Variation of different flow parameters: $(---)$ eddy viscocity $\varepsilon,(--$,$) fluid rms velocity in$ the $y$-direction (-) temperature profile $T-T_{\mathrm{w}}$. All parameters are non-dimensionalized with their respective values at $y^{+}=200$. 


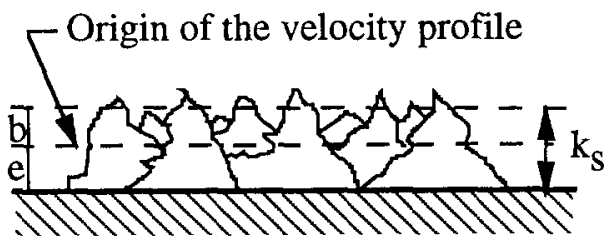

Fig. 4. Nomenclature for roughness parameters

Wood used the stopping distance model for estimating deposition velocity. We, however, use the unified deposition model, equation (22), for this purpose.

Finally, we account for the effect of "interception" by assuming that a particle is captured when its centre of mass is at a distance $r$ away from the effective roughness height. The lower limit of integration is taken as $y^{+}=y_{0}^{+}=y_{0} u_{*} / v$, where $y_{0}$ is given by

$$
y_{0}=0.45 k_{\mathrm{s}}+r
$$

The upper limit of integration is taken at $y^{+}=200$.

\section{RESULTS AND DISCUSSION}

Figures 5-9 are plots of non-dimensional deposition velocity versus non-dimensional relaxation time $\tau^{+}$. It should be recalled, however, that all equations of motion and calculations in this paper use the corrected relaxation time $\tau_{\mathrm{I}}$, given by equation $2(\mathrm{~b})$. $\tau_{1}$ includes corrections for large slip Reynolds number for large particles and Knudsen number correction for small particles. This is another novel and realistic feature of the present calculations not to be found in most previous calculations. It is only for plotting the results that the Stokes value $\tau^{+}$is used. This is done to be compatible with all previous theories and experimental results. Moreover, even for the same particle, the slip correction factor assumes different values at different points in the flow field as the local slip velocity varies. $\tau^{+}$, in the abscissa of the graphs, should be simply viewed as a non-dimensional representation of the particle size.

Figure 5 shows the relative importance of pure diffusion and pure inertial effects in the equation for mass flux [equation (22)]. For all curves in this figure, we assume that the effective roughness height, $k_{\mathrm{s}}$, is zero, and that the flow is isothermal (no thermal diffusion). The pure diffusion case is calculated by assuming that the turbulence is homogeneous. The source term in the RHS of equation (21) is zero and, consequently, the convective velocity, $\bar{V}_{\mathbf{p y}}^{\mathrm{c}+}$, is zero. Under these circumstances, equation (22) becomes identical with equation (6). As discussed after equation (9), the deposition velocity monotonically decreases with increasing relaxation time. This case was calculated by taking the lower boundary at $y^{+}=0$. The behaviour of the deposition velocity, however, changes if one includes the effects of interception. The lower boundary is now given by equation (23). As the lower boundary is shifted, the effective resistance against mass transfer tends to decrease. For large relaxation times, this effect can more than offset the effect of lower Brownian diffusion coefficient, $D_{\mathrm{B}}$. For large relaxation times, the calculated deposition velocity, therefore, increases substantially with increasing relaxation time (Fig. 5), even though the convective velocity, $\bar{V}_{\mathrm{p} y}^{\mathrm{c}}$, is neglected.

For calculating pure inertial effects, only the third term in the RHS of equation (22) is retained. Figure 5 shows that the convective velocity goes to zero for very small particles. Its effect on the deposition velocity has become comparable to that of pure diffusion around $\tau^{+} \sim 0.2$. It then rises steeply by several order of magnitude as $\tau^{+}$increases. The solid line in Fig. 5 is calculated by retaining all terms in equation (22). It merges with the pure diffusion case for very small particles and merges with the pure inertial case for large particles. The relative importance of diffusion, inertia and interception can clearly be appreciated from Fig. 5. 


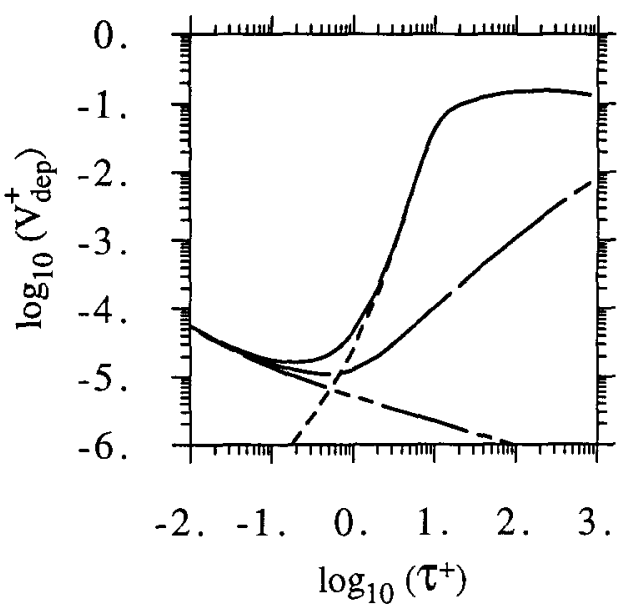

Fig. 5. Predicted deposition rate versus relaxation time (Effects of pure diffusion, pure inertia and interception). ( - Solution of equation (22) retaining all terms; (-- - ) Pure diffusion: solution of Fick's law, equation (6), with the lower boundary at wall $\left(y_{0}^{+}=0\right):(-\longrightarrow$ Pure diffusion: solution of Fick's law, equation (6), with interception $\left(y_{0}^{+}=r^{+}\right) ;(--\cdots--)$ Pure inertial deposition: solution of equation (22) retaining only the third term in the RHS. For all curves, $k_{s}^{+}=0, \Delta T=0$.

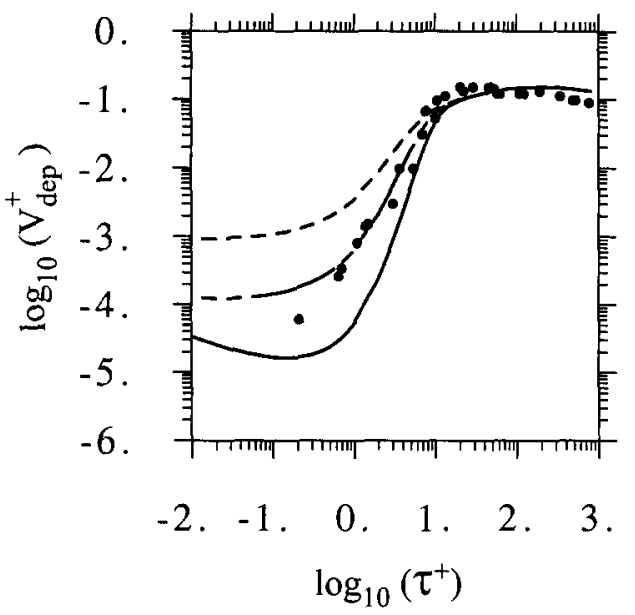

Fig. 6. Effects of surface roughness on the predicted deposition rate (without lift force) and comparison with experimental results of Liu and Agarwal (1974). ( $\stackrel{-}{-} k_{\mathrm{s}}^{+}=0,(--)_{\mathrm{s}}^{+}=0.5$, $(-\ldots) k_{\mathrm{s}}^{+}=1.5$. ( Experimental results of Liu and Agarwal. For all curves, $\Delta T=0$.

Figure 6 shows the variation in deposition velocity with relaxation time for three different roughness parameters: $k_{\mathrm{s}}^{+}=0, k_{\mathrm{s}}^{+}=0.5$ and $k_{\mathrm{s}}^{+}=1.5$. The Reynolds number and the density ratio $\left(\rho_{\mathrm{p}}^{0} / \rho_{\mathrm{f}}\right)$ are taken as 10,000 and 770 , respectively, as in Liu and Agarwal's experiments. Equations (21) and (22) are solved in full form for isothermal flow (no diffusion due to temperature gradient). The effect of roughness is reflected in the calculation procedure through a shift in the lower boundary of the computational domain. Expectedly, roughness affects the deposition velocity when diffusion is important. In the same figure the experimental data of Liu and Agarwal (1974) is also plotted for comparison. It can be seen that the present calculation scheme captures all the features of measured deposition velocity both qualitatively as well as quantitatively. Specifically, the calculated curve for $k_{\mathrm{s}}^{+}=0.5$ is almost superposed on the experimental values. Given that the deposition velocity varies by more than four order of magnitude in the range of investigation, and that it is calculated from a simple, universal equation [equation (22)], such agreement is indeed remarkable. 


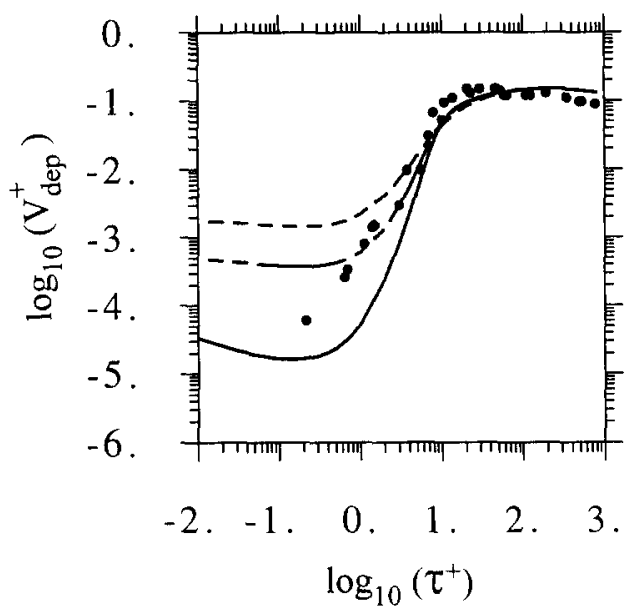

Fig. 7. Effects of diffusion due to temperature gradient on the predicted deposition rate (without lift force). ( $\longrightarrow \Delta T=0,(--) \Delta T=5 \mathrm{~K},(---) \Delta T=20 \mathrm{~K}$. (-) Experimental results of Liu and Agarwal. For all curves, $k_{s}^{+}=0$.

Figure 7 shows the effects of temperature gradient on the deposition velocity. Equations (21) and (22) are solved for three cases: $\Delta T=0, \Delta T=5 \mathrm{~K}$ and $\Delta T=20 \mathrm{~K}$, where $\Delta T$ is the temperature difference between the upper boundary of the calculation domain $\left(y^{+}=200\right)$ and the pipe wall (the wall is cooled). The roughness $k_{\mathrm{s}}^{+}$is assumed zero for all cases. Diffusion due to temperature gradient [second term in the RHS of equation (22)] is important for small particles. Even a small temperature difference (e.g. $\Delta T=5 \mathrm{~K}$ ) has a significant effect on the deposition velocity. For $1<\tau^{+}<10$, there is an interaction between thermophoresis and turbophoresis.

Some points regarding boundary conditions for equation (22) may be mentioned here. In the pure diffusion limit of very small particles, it is acceptable to set the concentration at the lower boundary at $y_{0}^{+}$to zero. However, in the pure inertial limit of large particles, a more appropriate condition at the lower boundary is $\partial \rho_{\mathrm{p}}^{+} / \partial y^{+}=0$. It was found, however, in the inertial limit, the effects of such boundary conditions on the magnitude of the calculated deposition velocity are negligible. This is because the convective velocity $\bar{V}_{\mathrm{py}}^{\mathrm{c}+}$ can be calculated from equation (21) without any reference to the concentration profile and in the inertial limit the deposition velocity is almost entirely controlled by $\bar{V}_{\mathrm{py}}^{\mathrm{c}}$. Although the deposition velocity remains unaffected, the concentration profile close to the wall obviously depends on the particular boundary condition employed. In the range of the intermediate particle sizes where both diffusion and particle inertia are important, it is envisaged that a boundary condition for particle concentration at the wall will possibly have some effects on the calculated deposition velocity. For a proper formulation of the boundary condition one would have to resort to kinetic theory.

Figure 8 shows the effects of Saffman lift force on the deposition velocity. As already explained, the inclusion of the lift force makes the $y$-momentum equation coupled with the $x$-momentum equation, and equations (16), (18a) and (18b) have to be solved simultaneously. The deposition velocity without lift force, as has been considered until now, is calculated from equations (16) and (18c). The lift force increases the deposition velocity especially in the particle size range where turbophoresis is the dominant mechanism. This is consistent with the findings of other authors, e.g. Kallio and Reeks (1989) and Fan and Ahmadi (1993). However, in this connection it should be noted that Saffman originally derived his result for unbounded shear flow whereas all of these works make the questionable use of the same expression for lift force in the vicinity of a solid wall.

Figure 9 shows the comparison of the present calculations with those of some other authors. The model of Friedlander and Johnstone (1957) gives the best agreement in the turbophoretic region. The difficulties of a stopping-distance model, however, have been discussed in Section 1.1. It is interesting to note that the predictions (with lift) of Fan and 


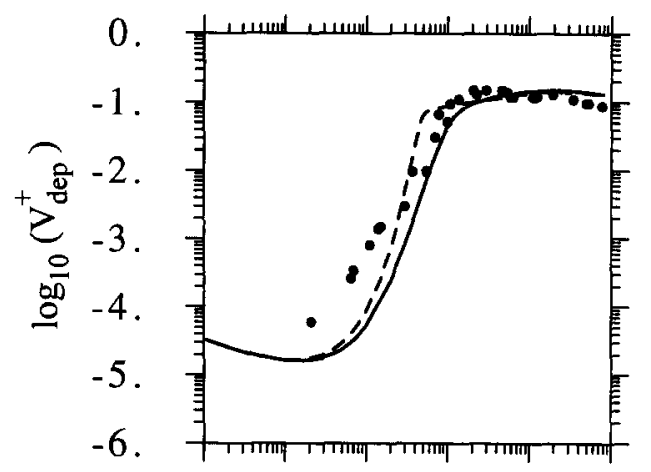

$$
\begin{array}{ccccc}
-2 . & -1 . & 0 . & 1 . & 2 . \\
\log _{10}\left(\tau^{+}\right) & &
\end{array}
$$

Fig. 8. Effects of Saffman lift force on the predicted deposition rate. (-) Without lift, (----) with lift. (-) Experimental results of Liu and Agarwal. For all curves, $k_{\mathrm{s}}^{+}=0, \Delta T=0$.

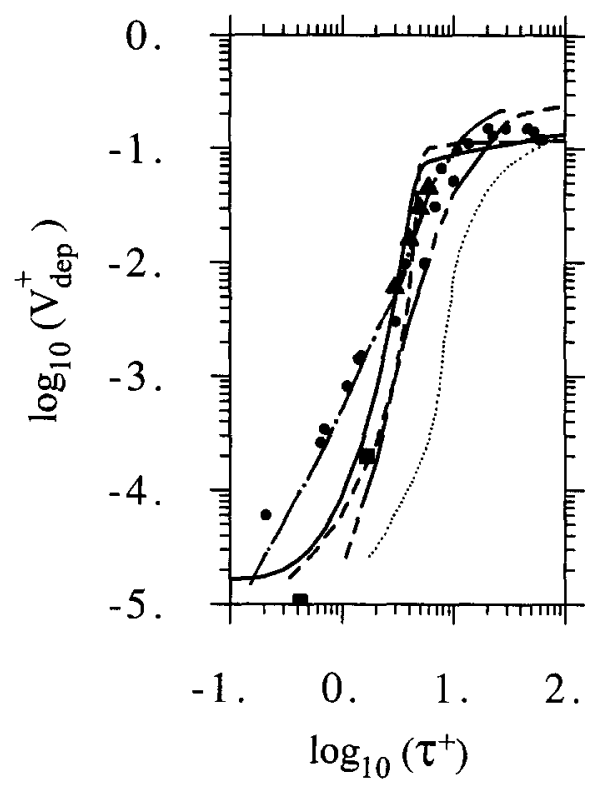

Fig. 9. Comparison of the present theory with experiments and predictions of various authors. (-) Experimental results of Liu and Agarwal. For all curves, $k_{\mathrm{s}}^{+}=0, \Delta T=0 .(-$ Present prediction (with lift), (-- -) Fan and Ahmadi (1993), (- - ) Fichman et al. (1988), (- - ) Friedlander and Johnstone (1957), ( . . . . . . ) Davies (1966). Direct numerical simulation: (\) McLaughlin (1989), (ם) Li and Ahmadi (1991).

Ahmadi (1993), who used a quite different approach of particle trajectory calculations involving the sublayer model of Cleaver and Yates, give quite similar results to the present calculations. (The density ratio $\left(\rho_{\mathrm{p}}^{0} / \rho_{\mathrm{f}}\right)$ is 770 in the present calculation, it was 965 in Fan and Ahmadi's calculation. The agreement is expected to have been better if both calculations used the same density ratio.) Fan and Ahmadi's calculations, on the other hand, tend to level off at a constant deposition rate for large particles. The deposition rate predicted by the present theory would decrease for large particles (Figs 6-8), as a result of the acceleration term (Section 4). This behaviour is consistent with experiments, and predictions of Reeks and Skyrme (1976) and Kallio and Reeks (1989). Good agreement is also observed with direct numerical simulation results of Mclaughlin (1989) and of Li and Ahmadi (1991) 
as quoted by Fan and Ahmadi (1993). The present analysis, however, is Eulerian, simple, fast and general. It is also quite flexible-equations (16)-(18), for example, already include the effects of thermophoresis, electrical forces, surface roughness, Saffman lift force and gravity, and could be easily extended to include other effects.

There is some discrepancy between the predictions and Liu and Agarwal's data for small particles. The experimental data compiled by McCoy and Hanratty (1977) and by Papavergos and Hedley (1984) show considerable scatter. We have plotted only Liu and Agarwal data as this is generally accepted as one of the most dependable data set. The aforementioned discrepancy is characteristic of other models also. Figure 9, for example, shows the predictions of Lagrangian models of Fan and Ahmadi (1993), Fichman et al. (1988), and, direct simulations of Li and Ahmadi (1991) quoted by Fan and Ahmadi (1993). Johansen (1991) also underpredicted by similar amounts and suggested that mirror charging could be responsible, although the value of the charge required for good agreement was found to be ten times the experimental value quoted by Liu and Agarwal. Wood's (1981) calculation suggested that a small amount of surface roughness $\left(k_{\mathrm{s}}^{+}=0.5\right)$ could account for the discrepancy. Figures 6 and 7 of the present study show that a small amount of roughness $\left(k_{\mathrm{s}}^{+}=0.5\right)$ or a small temperature gradient $(\Delta T<5 \mathrm{~K})$ produces very good agreement between the prediction and the experimental data. Further work on this issue is in progress.

The starting point of the present formulation has been Ramshaw's (1979) equations of motion which are internally consistent and physically satisfying. It is also very easy to include new forces in Ramshaw's equations thereby extending the scope of the theory of deposition. We have then systematically Reynolds-averaged both the continuity and momentum equations simultaneously. Finally, with minor approximations, we have converted equations (A3), (A6) and (A7) into the equation set (16)-(18) which is far simpler in form and is amenable to direct physical interpretation.

We have presented calculations for fully developed pipe flow. We have assumed that the pipe radius is large, so that we could write equations (16)-(18) in simple Cartesian coordinates. Similar equations can be derived in the cylindrical coordinates for axisymmetric, fully developed pipe flow. The analysis presented in Appendix A, until the condition for fully developed flow $\partial \bar{V}_{\mathrm{py}} / \partial x=0$ is invoked, is also applicable for the boundary layer type flow.

\section{CONCLUSIONS}

A unified theory of deposition is presented. The procedure consists of writing the particle continuity and momentum conservation equations and then conducting Reynolds averaging, which, for fully-developed vertical flow, ultimately results in the equation set (16), (18a) and (18b). The equations are simple, and a clear physical interpretation is possible for each term. The solution of these equations agrees well with experimental values of deposition velocity for the entire range of particle size. The method of Reynolds averaging is general, and other effects not included in this study e.g. pressure diffusion can easily be incorporated by including the appropriate forces in the particle momentum equation [equation (13)] as discussed.

The theory offers a simple, fast and reliable computational tool of practical use to aerosol engineers. The predictions are at least as accurate as the state-of-the-art particle-tracking calculations (Fig. 9) but require much less computational time. The theory includes the effects of thermophoresis, turbophoresis, electrostatic forces, gravity, Saffman lift force and surface roughness, and in this respect it is the most versatile theory that has appeared in the literature. The theory applies to particles of all sizes. (The particle relaxation time, equation (2b), includes correction for the departure from Stokesian drag regime for large particles and rarefied gas effects for very small particles. This is another novel realistic aspect of the calculations.) It is possible to combine the present scheme for calculating particle motion with well-established Eulerian flow solvers for calculating the flow field of the primary fluid. Thus, the present theory could be of interest to fluid dynamicists as well as of use to aerosol engineers. 
A great deal of physics can be learnt from and captured by the present simple analysis. The non-dimensionalized versions of the Reynolds averaged equations, equations (21) and (22), are used for calculating all results presented in this paper (except for Figs 8 and 9, where Saffman lift force is included and, consequently, equations (18a) and (18b) have to be solved together in place of equation (21)). The first term in the RHS of equation (22) is the contribution from Brownian and turbulent diffusion. This depends on the gradient of concentration and is identical to Fick's law, equation (6). The second term in the RHS of equation (22) represents the transport of particles as a result of temperature gradient. The third term in equation (22) represents a convective transport of particles resulting from the interaction of particle inertia with inhomogeneity of the fluid turbulence field. This term depends on the gradient of turbulence intensity. For small particles the first two terms dominate whereas for large particles it is the third term that matters (Fig. 5). That these three distinct mechanisms of deposition appear in a simple additive form in equation (22) has not been postulated but is rigorously derived from the fundamental conservation equations. Given that the deposition velocity varies by more than four order of magnitude in the range of particle sizes under investigation, and that it is calculated from a simple, universal equation (22) which contains only a modicum of empiricism and no "tuning factor", it is indeed remarkable that the calculated deposition velocity agrees so well with the experimental values.

It is crucial to incorporate the particle momentum equation [equation (21)] in the analysis. The absence of this equation in many previous analyses which solve only the particle continuity equation necessitated postulations such as the stopping-distance models. It is shown in Fig. 5 that, on the other hand, deposition velocity for particles bigger than a critical size could almost be determined from the momentum equation alone. (The critical size is about $\tau^{+}=1$ when $k_{\mathrm{s}}^{+}=0$ and $\Delta T=0$ as shown in Fig. 5, but increases when thermal diffusion or roughness elements are present as shown in Figs 6 and 7.) The first term in equation (21) represents particle acceleration, the second term is the viscous drag and the third term arises from the turbulent fluctuations. When $\tau^{+} \rightarrow \infty$, the viscous drag term is negligible, and the acceleration term is balanced by the turbulence term. However, in this limit, the turbulence term also tends to zero as $\Re \rightarrow 0$. As $\tau^{+}$is decreased from this limit, the turbulence term grows and so does the convective slip velocity $\bar{V}_{\mathrm{p} y}^{\mathrm{c}+}$. The deposition velocity therefore increases with decreasing particle size in this range (Fig. 1, zone 3). This trend in deposition velocity, however, does not continue all the way to very small particles because the viscous drag term assumes importance. The viscous drag term increases with decreasing particle relaxation time, and tries to reduce the slip velocity. The turn over point occurs around $\tau^{+}=30$. For $\tau^{+}<30$, the deposition velocity starts decreasing with decreasing relaxation time (Fig. 1, zone 2). In this regime, the acceleration term loses importance, and the viscous term usually balances the turbulence term. Equation (21) shows that as $\tau^{+} \rightarrow 0$, $\mathfrak{R} \rightarrow 1$ and $\bar{V}_{\mathrm{py}}^{\mathrm{c}+} \rightarrow 0$. Turbophoresis is thus negligible for small particles even if there is a gradient in turbulence intensity. However, for small particles the Brownian and turbulent diffusion of particles begin to dominate and the deposition velocity rises again with decreasing $\tau^{+}$(Fig. 1, zone 1).

It is to be noted that it is because of the acceleration term in equation (21) that the deposition velocity decreases with increasing relaxation time in zone 3 of Fig. 1. If the acceleration term was not included, equation (21) would have predicted a constant deposition velocity for very large relaxation times [the predictions of Fan and Ahmadi (1993) show this behaviour for $\left.\tau^{+}>10\right]$. This is so because, as $\tau^{+} \rightarrow \infty$, although $\Re \rightarrow 0$, the product $\tau^{+} \mathfrak{R}$, however, remains finite in this limit. Equation (20) shows that, as $\tau^{+} \rightarrow \infty$, $\tau^{+} \mathfrak{R} \rightarrow 1.43 T_{\mathrm{L}}^{+}$.

Present calculations (Fig. 6) show that the presence of small surface roughness even in the hydraulically smooth regime significantly enhances deposition of small particles. Figure 7 shows that thermophoresis can be equally important (and should be considered, for example, in deposition calculations for internally cooled gas turbine blades). For intermediate size particles, there can be a strong interaction between thermophoresis and turbophoresis. 
The effects of different deposition mechanisms come out naturally from the present analysis in a physically satisfying manner and there is scope to add other effects in a straightforward, logical way. The present theory is also logical in finding the combined effects of different deposition mechanisms, as the appropriate forces are added in the momentum equation and the combined "velocity" or flux is calculated by solving the continuity and momentum equations. This should be superior to the often-used linear addition of respective "velocities" in order to determine the combined mass flux (e.g. adding a turbophoretic velocity with a diffusive velocity).

A particular turbulence model has been used here (Section 2.5) for illustration, but the present theory is not limited on this account. Any other suitable turbulence model for describing the fluid flow field could be used in conjunction with the present theory of deposition. Similarly, for all numerical illustrations and for comparisons with experiments and other theoretical predictions, we have considered the most explored flow configuration: vertical, fully-developed pipe flow. However, the analysis presented in Appendix A, until the condition for fully developed flow $\partial \vec{V}_{\mathrm{p} y} / \partial x=0$ is invoked, is also applicable for the boundary-layer-type flow. Equations (16)-(18) are almost exact and should work well if the particle RMS velocity could be accurately predicted as a function of wall coordinate and particle relaxation time. Given the success of the present simple scheme, further research is intended to improve on this aspect.

Acknowledgement-I am indebted to Gonville and Caius College, Cambridge, for electing me as a Fellow and to Dr. J. B. Young for his help. A part of this work was completed at the Aerospace Engineering Department, University of Bristol.

\section{REFERENCES}

Beal, S. K. (1970) Deposition of particles in turbulent flow on channel or pipe walls. Nucl. Sci. Engng 40, 1-11. Binder, J. L. and Hanratty, T. J. (1991) A diffusion model for droplet deposition in gas/liquid annular flow. Int. $J$. Multiphase Flow 17, 1-11.

Brooke, J. W., Hanratty, T. J. and McLaughlin, J. B. (1994) Free-flight mixing and deposition of aerosols. Phys. Fluids 6, 3404-3415.

Cleaver, J. W. and Yates, B. (1975) A sub layer model for the deposition of particles from a turbulent flow. Chem. Engng Sc. 30, 983-992.

Davies, C. N. (1966) In Aerosol Science (Edited by Davies, C.N.). Academic Press, London.

Fan, F. and Ahmadi, G. (1993) A sublayer model for turbulent deposition of particles in vertical ducts with smooth and rough surfaces. J. Aerosol Sci. 24, 45-64.

Fichman, M., Gutfinger, C. and Pnueli, D. (1988) A model for turbulent deposition of aerosols. J. Aerosol Sci. 19, 123-136.

Friedlander, S. K. and Johnstone, H. F. (1957) Deposition of suspended particles from turbulent gas streams. Ind. and Engng Chem. 49, 1151-1156.

Guha, A. (1995) Two-phase flows with phase transition. VKI Lecture Series 1995-06 (ISSN0377-8312), pp. 1-110. von Karman Institute for Fluid Dynamics.

Johansen, S. T. (1991) The deposition of particles on vertical walls. Int. J. Multiphase Flow 17, 355-376.

Kallio, G. A. and Reeks, M. W. (1989) A numerical simulation of particle deposition in turbulent boundary layers. Int. J. Multiphase Flow 15, 433-446.

Kay, J. M. and Nedderman, R. M. (1988) Fluid Mechanics and Transfer Processes. Cambridge University Press. Cambridge.

Liu, B. Y. H. and Agarwal, J. K. (1974) Experimental observation of aerosol deposition in turbulent flow. J. Aerosol Sci. 5, 145-155.

Liu, B. Y. H. and Ilori, T. Y. (1974) Aerosol deposition in turbulent pipe flow. Environ. Sci. Technol. 8, 351-356.

McCoy, D. D. and Hanratty, T. J. (1977) Rate of deposition of droplets in annular two-phase flow. Int. J. Multiphase Flow 3, 319-331.

McLaughlin, J. B. (1989) Aerosol particle deposition in numerically simulated channel flow. Phys. Fluids 1, $1211-1224$.

Ounis, H., Ahmadi, G. and McLaughlin, J. B. (1993) Brownian particle deposition in a directly simulated channel flow. Phys. Fluids 5, 1427-1432.

Papavergos, P. G. and Heddley, A. B. (1984) Particle deposition behaviour from turbulent flows. Chem. Engng. Res. Des. 62, 275-295.

Ramshaw, J. D. (1979) Brownian motion in a flowing fluid. Phys. Fluids. 22, 1595-1601.

Reeks, M. W. and Skyrme, G. (1976) The dependence of particle deposition velocity on particle inertia in turbulent pipe flow. J. Aerosol Sci. 7, 485-495.

Reeks, M. W. (1983) The transport of discrete particles in inhomogeneous turbulance. J. Aerosol Sci. 14, 729-739.

Reeks, M. W. (1992) On the continuum equations for dispersed particles in nonuniform flows. Phys. Fluids 4, 1290-1303. 
Talbot, L., Cheng, R. K., Schefer, R. W. and Willis, D. R. (1980) Thermophoresis of particles in a heated boundary layer. J. Fluid Mech. 101, 737-758.

Vames, J. S. and Hanratty, T. J. (1988) Turbulent dispersion of droplets for air flow in a pipe. Exper. Fluids 6, 94-104.

Wood, N. B. (1981) A simple method for the calculation of turbulent deposition to smooth and rough surfaces. J. Aerosol Sci. 12, 275-290.

Young, J. B. (1991) User's manual for computer program PARTICLE, Private communication.

\section{APPENDIX A: DETERMINATION OF THE PARTICLE FLUX BY REYNOLDS AVERAGING OF THE PARTICLE CONTINUITY AND MOMENTUM EQUATIONS}

Figure A1 shows the cartesian co-ordinate system used. Substitute equation (15) in equations (12)-(13) and then take time-mean of the resulting equations. We neglect the $x$-variation of any Reynolds stress terms and triple correlations of primed quantities. The continuity equation [equation (12)] then becomes,

$$
\frac{\partial}{\partial x}\left(\bar{\rho}_{\mathbf{p}} \bar{V}_{\mathbf{p} x}\right)+\frac{\partial}{\partial y}\left(\bar{\rho}_{\mathbf{p}} \bar{V}_{\mathrm{p} y}+\overline{\rho_{\mathrm{p}}^{\prime} V_{\mathrm{p} y}^{\prime}}\right)=0 .
$$

Equation (A1) shows that the particle mass flux in the $y$-direction, $J$, is given by

$$
J=\bar{\rho}_{\mathrm{p}} \bar{V}_{\mathrm{p} y}+\overline{\rho_{\mathrm{p}}^{\prime} V_{\mathrm{p} y}^{\prime}} \text {. }
$$

The Reynolds-averaged $y$-momentum equation [equation (13)] can be written as

$$
\begin{aligned}
& \frac{\partial}{\partial x}\left(\bar{\rho}_{\mathbf{p}} \bar{V}_{\mathrm{p} x} \bar{V}_{\mathrm{p} y}\right)+\frac{\partial}{\partial y}\left(\bar{\rho}_{\mathbf{p}} \bar{V}_{\mathrm{p} y} \bar{V}_{\mathrm{p} y}\right)+\frac{\partial}{\partial y}\left(\bar{\rho}_{\mathrm{p}} \overline{V_{\mathrm{p} y}^{\prime 2}}\right)+2 \frac{\partial}{\partial y}\left(\bar{V}_{\mathrm{p} y} \overline{\rho_{\mathrm{p}}^{\prime} \bar{V}_{\mathrm{p} y}^{\prime}}\right) \\
& =\frac{1}{\tau_{1}}\left(-D_{\mathrm{B}} \frac{\partial \bar{\rho}_{\mathrm{p}}}{\partial y}-D_{\mathrm{T}} \bar{\rho}_{\mathrm{p}} \frac{\partial \ln T}{\partial y}+\bar{\rho}_{\mathrm{p}} \tau_{\mathrm{I}}\left(F_{s y}+G_{\mathrm{E} y}\right)+\bar{\rho}_{\mathrm{p}}\left(\bar{V}_{\mathrm{f} y}-\bar{V}_{\mathrm{p} y}\right)+\overline{\rho_{\mathrm{p}}^{\prime} V_{\mathrm{f} y}^{\prime}}-\overline{\rho_{\mathrm{p}}^{\prime} V_{\mathrm{p} y}^{\prime}}\right)+\bar{\rho}_{\mathrm{p}} g_{y},
\end{aligned}
$$

where $G_{\mathrm{E} y}$ is the $y$-component of electrostatic force per unit mass on the particles and $g_{y}$ is the component of the gravitational acceleration in the $y$-direction. The coefficient of diffusion due to temperature gradient, $D_{T}$, is given by $D_{T}=D_{\mathbf{B}}(1+\eta / k T)$, and $F_{S}$, the Saffman lift force in the $y$-direction, is given by

$$
F_{\mathrm{S} y}=1.542 \frac{\rho_{\mathrm{f}}}{\rho_{\mathrm{p}}^{0}} v \frac{1}{r} \sqrt{\frac{1}{v}\left|\frac{\mathrm{d}}{\mathrm{d}} \overline{\mathrm{V}}_{\mathrm{f} x}\right|}\left(\bar{V}_{\mathrm{f} x}-\bar{V}_{\mathrm{p} x}\right) .
$$

We now make some simplifications of (A3). The average $y$-component of fluid velocity is zero, i.e., $\bar{V}_{\mathrm{f} y}=0$. In fully developed pipe flow $\hat{\partial} \vec{V}_{\mathrm{p} y} / \partial x=0$. The particle mass flux due to turbulent fluctuation is modelled by gradient diffusion

$$
\overline{\rho_{\mathrm{p}}^{\prime} V_{\mathrm{p} y}^{\prime}}=-D_{\mathrm{t}} \frac{\partial \bar{\rho}_{\mathrm{p}}}{\partial y}
$$

where $D_{1}$ is the mass diffusivity of the particles due to turbulence and usually taken as the eddy viscosity of the fluid, $\varepsilon$. We further assume that

$$
\overline{\rho_{\mathrm{p}}^{\prime} V_{\mathrm{f} y}^{\prime}}-\overline{\rho_{\mathrm{p}}^{\prime} V_{\mathrm{py}}^{\prime}} \approx \tau_{\mathbf{1}} \overline{V_{\mathrm{py}}^{\prime 2}} \frac{\partial \bar{\rho}_{p}}{\partial y} \text {. }
$$

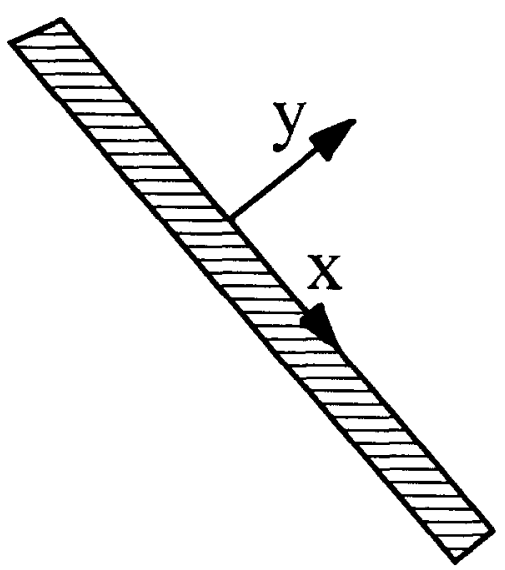

Fig. A1. Co-ordinate system used for calculation of deposition on a solid surface. 
Consider the special case of vertical flow, i.e. the co-ordinate $x$ is in the vertical direction. $g$ then acts wholly in the $x$-direction and $g_{y}$ is zero. Substitution of equations (A1). (A4), (A5), and the conditions $\bar{V}_{f y}=0$ and $\partial \bar{V}_{\mathrm{py}} / \hat{\partial} x=0$ in equation (A3) results in,

$$
\begin{aligned}
& \left(J-D_{\mathrm{t}} \frac{\partial \bar{\rho}_{\mathrm{p}}}{\partial y}\right) \frac{\partial \bar{V}_{\mathrm{p} y}}{\partial y}+\frac{\bar{\rho}_{\mathrm{p}} \bar{V}_{\mathrm{p} y}}{\tau_{\mathrm{I}}}-\bar{V}_{\mathrm{p} y} \frac{\partial}{\partial y}\left(D_{1} \frac{\partial \bar{\rho}_{\mathrm{p}}}{\partial y}\right) \\
& =\frac{1}{\tau_{1}}\left(-D_{13} \frac{\partial \bar{\rho}_{\mathrm{p}}}{\partial y}-D_{\mathrm{\Gamma}} \bar{\rho}_{\mathrm{p}} \frac{\hat{\partial} \ln T}{\partial y}-\tau_{1} \bar{\rho}_{\mathrm{p}} \frac{\partial \overline{\bar{V}_{\mathrm{py}}^{2}}}{\partial y}+\tau_{1} \bar{\rho}_{p}\left(F_{\mathrm{Sy}}+G_{\mathrm{Fy}}\right)\right) .
\end{aligned}
$$

The correspondingly simplified Reynolds-averaged $x$-momentum conservation equation for the particles is given by

$$
J \frac{\hat{\partial} \bar{V}_{\mathrm{px}}}{\hat{\partial} y}-\frac{\hat{\partial}}{\hat{\partial} y}\left(\bar{\rho}_{\mathrm{p}} D_{\mathrm{t}} \frac{\hat{\imath} \bar{V}_{\mathrm{p} x}}{\hat{\partial} y}\right)=\frac{\bar{\rho}_{\mathrm{p}}}{\tau_{\mathrm{I}}}\left(\bar{V}_{\mathrm{r} x}-\overline{\boldsymbol{V}}_{\mathrm{p} x}\right)+\bar{\rho}_{\mathrm{p}} g
$$

where $g$ is the acceleration due to gravity, and the turbulence term $\overline{V_{p x}^{\prime} V_{p y}^{\prime}}$ has been modelled by $V_{\mathrm{p} x}^{\prime} V_{\mathrm{p} y}^{\prime}=-D_{\mathrm{t}}\left(\hat{c} \bar{V}_{\mathrm{p}} / \hat{\sigma} y\right)$. In the general case, equations $(A I)$. (A2), (A6) and $(A 7)$ are to be solved simultaneously to determine $\vec{\rho}_{\mathrm{p}}, \vec{V}_{\mathrm{p} x}$ and $\bar{V}_{\mathrm{p}: \mathrm{x}}$

Note that $x$-momentum equation (A7) involves both $\bar{V}_{p x}$ and $\bar{V}_{p y}$. The $v$-momentum equation (A6), on the other hand, is almost decoupled and depends on $\bar{V}_{\mathrm{p} x}$ only through the Saffman lift force, $F_{\mathrm{S}_{\mathrm{y}}}$. In the general case, as indicated earlier, one could retain all terms in equations (A6) and (A7), and solve simultaneously. Our aim, however, is to develop a simple theory of deposition that brings out the underlying physics. For simplification, the third term in the LHS of equation (A6). containing the second derivative of the concentration profile, is neglected. We consider the absolute particle velocity to be the sum of a diffusive and a convective part. We define the convective velocity of the particles, $V_{\mathrm{p}}^{\mathrm{c}}$, by

$$
\mathbf{V}_{\mathrm{p}}=\mathbf{V}_{\mathrm{p}}^{\mathrm{c}}-\left(D_{\mathrm{B}} \nabla\left(\ln \rho_{\mathrm{p}}\right)+D_{T} \nabla(\ln T)\right) .
$$

$\mathbf{V}_{\mathrm{p}}$ is very close to $\mathbf{V}_{\mathrm{p}}^{\mathrm{c}}$ for large particles. For smaller particles $\left(\tau^{+}<10\right)$, the acceleration term (the first term in the LHS of equation (A6) itself is not significant. The acceleration term in equation (A6) can, therefore. be approximated by $\bar{V}_{\mathrm{py}}^{\mathrm{c}} \hat{\mathrm{V}} \bar{V}_{\mathrm{py}}^{\mathrm{c}} / \hat{\mathrm{C} y}$. With these simplifications and with the help of (A8), equation (A6) can be rewritten as

$$
\bar{V}_{\mathrm{py}}^{\mathrm{c} y} \frac{\partial \bar{V}_{\mathrm{py}}^{\mathrm{c}}}{\partial y}+\frac{\bar{V}_{\mathrm{p} y}^{\mathrm{c}}}{\tau_{1}}=-\frac{\overline{\partial V_{\mathrm{p} y}^{\prime 2}}}{\partial y}+\mathbf{F}_{S_{y}}+G_{\mathrm{E} y}
$$

Equation (A9) is almost exact and do not depend on the concentration profile. If $F_{\mathrm{S}}$ is not considered, equation (A9) is a first order, non-linear differential equation involving only one unknown. $\vec{V}_{\mathrm{p} \text { y }}^{\mathrm{c}}$. It can be written in finite difference form and integrated with one boundary condition $\left(\vec{V}_{\mathrm{pr}}^{\mathrm{s}}=0\right.$ at the pipe centreline, or, at a sufficient distance away from the wall, say at $y^{+}=200$, where the gradient in turbulence intensity is negligibly small). The convective velocity, $\vec{V}_{\mathrm{pm}}^{\mathrm{e}}$, can, therefore, be determined if the particle RMS velocity could be estimated properly. For not so large particles, the drag term (second term in LHS) almost balances the turbulence term (first term in RHS), and, the acceleration term (first term in LHS) is negligible. The acceleration term assumes importance for large particles.

Similar to (A9), equation (A7) can be simplified to

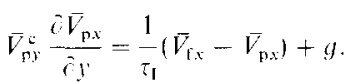

Finally, equations (A2). (A4) and (A8) can be combined to give the expression for particle flux,

$$
\begin{aligned}
J & =\bar{\rho}_{\mathrm{p}} \bar{V}_{\mathrm{p} y}+\overline{\bar{\rho}_{\mathrm{p}}^{\prime} V_{\mathrm{p} y}^{\prime}} \\
& =-\left(D_{\mathrm{B}}+D_{\mathrm{v}}\right) \frac{\hat{\rho} \bar{\rho}_{\mathrm{p}}}{\hat{\partial} y}-D_{T} \bar{\rho}_{\mathrm{p}} \frac{\hat{\gamma} \ln T}{\partial y}+\bar{\rho}_{\mathrm{p}} \bar{V}_{\mathrm{p} y}^{\mathrm{c}} .
\end{aligned}
$$

The equation sets $[(\mathrm{A} 1),(\mathrm{A} 2),(\mathrm{A} 6),(\mathrm{A} 7)]$ and $[(\mathrm{A} 9)-(\mathrm{A} 11)]$ are almost equivalent. The latter has the advantage that it is far simpler in form and each term has a clear physical interpretation.

\section{APPENDIX B: THE ROLE OF GRAVITY IN DETERMINING THE DEPOSITION VELOCITY IN FULLY DEVELOPED VERTICAL FLOW}

A study of equations (18a) and (18b) shows nicely how, in a vertical flow, gravity affects the $y$-momentum equation through the Saffman lift force. The LHS of equation (18b) involves $\bar{V}_{\mathbf{p} \text {. }}^{\mathrm{c}}$. As a result of this convective velocity, the direction of the lift force may remain unaltered whether the flow is vertically downwards or upwards [for upward flow replace $g$ by $-g$ in equation (18b)].

Consider the expression for the Saffman lift force given in Appendix A. The sign of the lift force depends on whether the particle velocity in the $x$-direction leads or lags the local fluid velocity.

$$
\operatorname{Sign}\left\{F_{\mathrm{Sy}}\right\}=\operatorname{Sign}\left\{\vec{V}_{\mathrm{f} x}-\vec{V}_{\mathrm{p}, x}\right\} \text {. }
$$

In a vertical downward flow $\bar{V}_{p x}>\bar{V}_{I_{x}}$ because of gravitational settling. Equation (B1) then shows that the Saffman lift force operates in the negative $y$-direction. i.e. towards the solid wall. Thus, Saffman lift force enhances the deposition velocity in this case (see Fig. 8). 


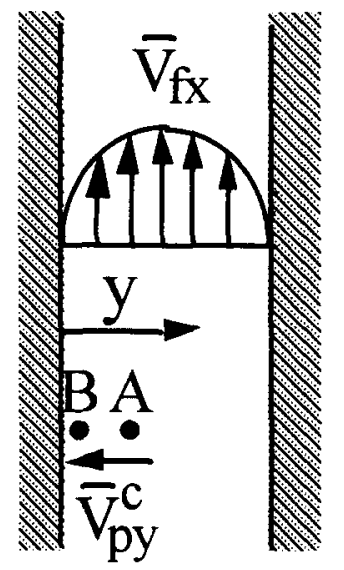

Fig. B1. Fully developed verticle upward flow.

In a fully developed vertically upward flow gravitational settling would tend to make $\bar{V}_{\mathrm{p} x}$ lag the fluid velocity. However, the particle convective velocity $\bar{V}_{\mathrm{p} y}^{\mathrm{c}}$ may alter the situation. Consider Fig. B1. $\bar{V}_{\mathrm{f} x}$ decreases from the centre of the flow passage towards the solid wall because of frictional effects, since the no-slip condition demands zero velocity at the solid wall. Thus, a particle arriving from higher (say, point A in Fig. B1) to a lower (say, point B) $y$-location may have higher $x$-velocity than the local fluid $x$-velocity [depending on the relative magnitudes of the gravitational settling velocity and the particle convective velocity $\left.\bar{V}_{\mathrm{py}}^{\mathrm{c}}\right]$. Consequently, it is still possible that the Saffman lift force would act towards the solid wall and enhance the deposition velocity even in vertically upward flow. However, it is expected that, other conditions remaining the same, the deposition velocity would be lower in upward than in downward flow of the fluid.

The above discussion is valid for fully developed vertical channel flow, in which the sole cause of the "laminar slip" between the particles and the fluid is gravity. In a general flow field, the "laminar slip" may result from the inability of the particles to follow curved streamlines exactly. 